\title{
The Extent and Factors Associated with Non-Prescription Medicine Use in Eastern India - A Cross-Sectional Survey
}

\author{
Manisha Das', Supriyo Choudhury', Ranjita Santra (Dhali) ${ }^{2 *}$, Rituparna De ${ }^{1}$, Ranendra Kumar Roy ${ }^{1}$ \\ 'Department of Pharmacology, College of Medicine \& Sagore Dutta Hospital, Kolkata-700058, West Bengal, INDIA. \\ 2Department of Clinical and Experimental Pharmacology, Calcutta School of Tropical Medicine, Kolkata-700073, West Bengal, INDIA.
}

\begin{abstract}
Objective: To assess the extent, pattern and determinants of non-prescription medicine use in an urban area of eastern India. Methods: A descriptive cross-sectional survey with total 392 subjects was carried out for 3 months by a structured questionnaire to assess the extent, pattern and determinants of non-prescription medicine use amongst the patients at a community retail medicine shop and a pharmacy running in Public-Private Partnership (PPP) model in a government hospital. Results: Our study found that $61.4 \%$ of the consumers indulged in the practice of self-medication. The commonest reason for self medication was a prevailing tendency in the community followed by cost-saving and convenience. The most commonly used medicines were antacids (43.4\%) followed by analgesics-antipyretics (42.6\%). It was found that only $12.5 \%$ completed an ongoing course of antibiotics. Consumption of ORS was commoner in diarrhoea than vomiting and only a few (13\%) of the patients dissolved the ORS powder as directed. Only $9.7 \%$ of the subjects thought non-prescription medicine use is safe. Regarding the various types of medicine preparations used by them from the two types of pharmacy, a significant difference was noted only for eye drops $(p=0.003$ ). This result might have been obtained due to prevailing ocular infections in the selected study population and underreporting at the Ophthalmology OPD of the nearest hospital. Conclusion: The current
\end{abstract}

study has documented the extent of, factors associated with, and the pattern of non-prescription medicine use resulting in a surge of self-medication practice in urban area.

Key words: Non-prescription medicine use, Self-medication, PPP model, Retail pharmacy, Survey.

Key message: Given the increasing emphasis on self-care and empowering the public to manage their health with non-prescription medicines, the findings highlight the need for improved pharmacovigilance of these medicines to maximize benefits with minimal risk. Healthcare providers need to be aware of the potential for misuse, abuse and dependence, particularly in patients with long-term illness.

Correspondence :

Ranjita Santra (Dhali), Assistant Professor, Department of Clinical and Experimental Pharmacology, Calcutta School of Tropical Medicine, 108, Chittaranjan Avenue, Kolkata-700073, West Bengal, INDIA.

Phone: 09836105240

Email: dsdrranjita@gmail.com

DOI: 10.5530/jyp.2017.9.111

\section{INTRODUCTION}

Irrational use of medicine along with self-medication poses a great problem amongst the medicine prescribers. Self medication can be defined as obtaining and consuming medicines without the advice of a physician either for diagnosis, prescription or surveillance of treatment. ${ }^{1}$ Self-medication is widely practiced worldwide and often considered as a component of self-care. ${ }^{2}$ It has both advantage and disadvantages. The World Health Organization (WHO) has appropriately pointed out that responsible self-medication can help prevent and treat diseases that do not require medical consultation. It also provides a cheaper alternative for treating common illnesses. ${ }^{3}$ On the contrary non-prescription medicine use in our country might lead to wastage of resources, increased resistance of pathogens, serious health hazards such as adverse medicine reactions and prolonged morbidity. Inadequate access to health care, lack of proper reinforcement of regulatory control and easy availability of medicines without prescription are among many factors responsible for non-prescription medicine use in our country. In the recent last, the US-FDA has restricted 46 new medicines from over the counter. Hence, various studies are being conducted targeting self medication. A recent study from Malaysia has shown that self-medication occurred with an average of 1.3 medicines per participant for GI and metabolic disorders (31.8\%) followed by respiratory conditions (19.4\%) and musculoskeletal (15.8\%). ${ }^{4}$ Another study found that self-medication was preferred by $87 \%$ of the study participants. The common reasons for self-medication were the ailments being minor $(85.2 \%)$ or prior experience with the same condition (49.5\%). In $84.4 \%$ responders, community pharmacists provided advice for self-medication. ${ }^{5}$ A study on the prevalence of self medication practice among the university students of South-western Nigeria has shown that the practice of self-medication was higher among the age group of $25-44$ years but lower in the $15-24$ and $\geq 45$ years age groups, respectively and where females exhibited greater practice of self-medication. Among undergraduate students, self-medication increased as the students' class level in the university increased. Postgraduate students exhibited low prevalence of self-medication practices. ${ }^{6}$ Two previous studies were conducted in China on knowledge, attitude and behaviours of university students on the use of antibiotics. The common indications of antibiotic use were common cold, sore throat, and fever. It was seen that during treatment course students switched to other antibiotic, and also changed the dosing strength and frequency. Majority of the students failed to complete the course of antibiotic. In both the studies adverse drug reactions (ADRs) were noted in about $16 \%$ of cases. ${ }^{7,8} \mathrm{~A}$ recent study was done in Europe for self-medication awareness for antibiotics. The major sources of procurement of the antibiotics were the community pharmacies and left-over medicines bought previously, easy availability of antibiotics without prescription, pack based dispensing system, and misconception about efficacy for minor illness of these class of medicines were found to be potential formidable factors. ${ }^{9}$ In a study conducted in Israel by Vaknin S et al., only $15 \%$ of the non prescription medicine users thought that practice of self-medication

This is an open access article distributed under the terms of the Creative Commons Attribution-NonCommercial-ShareAlike 4.0 License, which allows others to remix, tweak, and build upon the work non-commercially, as long as the author is credited and the new creations are licensed under the identical terms. 
is not safe. The factors are personal experience of purchaser, followed by pharmacist's recommendation, ${ }^{10}$ Anraku $\mathrm{M}$ et al performed a research in Fukuyama among elderly people and children to estimate the extent of use of over-the-counter (OTC) medicines and health food. In children, the most frequently consumed OTC medicines were cold medicines (32.1\%), followed by topical creams (22.6\%) and eye lotion (14.3\%). In elderly people, the most frequently consumed OTC products were eye lotion (18.0\%), followed by laxatives (14.8\%) and fomentation agents (13.1\%). ${ }^{11}$ Nielsen MW et al had undertaken a study to analyze the association among different types of medicine use and different measures of socio-economic position (SEP) in one and the same general population. It was found that pensioner and self employed use less OTC than salaried employees. Low income consumes less OTC medicines than high income group. Significant difference was not associated with gender and education. ${ }^{12}$ Yeufeng L et al had shown the pattern of use and factors associated with self-treatment in China. The study reveals that the prevalence of self-treatment with a recall period of two-weeks were significantly higher in urban compared with rural areas. ${ }^{13}$ Literature search also revealed that among the medical factors, the most important was medicine safety, followed by efficacy, contraindications, indications, and side effects. Among the business factors, the most important were brand and price. ${ }^{14}$ Very few studies have been conducted in India in this regard especially in the Eastern part of India. The study in 2014 in a rural area of north India 15 showed that $50 \%$ of patients were seeking self-medication for headache and other types of pain (23.3\%), fever (14.5\%), UTI (9.7\%) and RTI (11.7\%). The medicines most commonly purchased for practicing self-medication were NSAIDs (25.3\%), medications used for gastrointestinal disorders (20.8\%) and antibiotics (16.7\%). Prevalence of self-medication was higher primarily among illiterate males aged above 15 years with annual low income. ${ }^{15}$ Another research reflected similar sources of information about medicines. $67.3 \%$ procured their medicines from medicine shops and the rest from left over medicines. An Indian study was conducted in Hyderabad and Secundrabad on the non-prescription profile of medicine use in rural and urban population. It was found to be higher in the urban areas (37\%) as compared to rural areas (17\%). Nutritional products, compounds with analgesic, antipyretic and antiinflammatory effects, and broad spectrum antibiotics constituted a high proportion of non-prescription medicines used in both urban and rural areas. ${ }^{17}$

Therefore, the objectives of this study were to assess the extent, pattern and determinants of non-prescription medicines use in an urban area of eastern India.

\section{MATERIALS AND METHODS}

This questionnaire based descriptive cross-sectional study was conducted amongst consumers from a retail medicine shop in the community and a pharmacy running in PPP model in a government hospital premises in Kolkata between June-July, 2014. Questionnaire development was done by literature search and also inputs from the researchers with expertise in this area. Among the consumers of the medicine outlets, the nonprescription medicine users were included in this study after obtaining written informed consent. The study subjects were male or female aged between 15-75 years and either patient or patient parties or other persons buying medicines from that PPP pharmacy and retail medicine shop. Sampling strategy applied in this study was purposive. Persons who bought medicines regularly for a chronic illness like essential hypertension, hypothyroidism, diabetes, and neurological disorders were excluded from the study. Persons who were unable to read or write in any of the 3 languages viz. English, Bengali and Hindi were also excluded from this study. Institutional Ethics Committee (IEC) permission was obtained prior to the initiation of the data collection.
The non-prescription medicine users were given a validated multiple response type questionnaire with both open and closed ended questions to analyze the pattern of different medicines used by them and the factors associated with such use. The questionnaire was supplied in any of 3 languages viz. English, Bengali and Hindi depending on the acceptability to the subject. We approached 638 medicine consumers in the community that was selected for this study and out of them 392 (61.4\%) were found to be non- prescription medicine users, of whom 352 subjects had given informed consent to participate, with a non-responder rate of $10 \%$. Calculation of sample size was done from online survey sample size calculator website, Raosoft (http://www.raosoft.com/samplesize.html), considering a margin of error of 5\%, a 95\% confidence level. The population of Kolkata was considered over 14 million (including residents and immigrants).

\section{Statistical analysis}

Data were analyzed using SPSS Version 20 software. Summary statistics were expressed using mean and standard deviation (SD) for numerical variables (median and interquartile ranges when skewed) and counts and percentages for categorical variables. Numerical variables were compared between generic and branded medicine users using Student's $\mathrm{t}$ test when normally distributed and Mann-Whitney U test when skewed. Fisher's exact test was employed for intergroup comparison of categorical variables. Comparisons were two-sided and $p<0.05$ was taken to be statistically significant.

\section{RESULTS}

The proportion of non-prescription medicine users in the two study groups i.e. from the retail medicine shop was $66.8 \%$ (246 out of 368 ) and another a pharmacy running in PPP model in a government hospital premises in Kolkata is $54 \%$ ( 146 out of 270 ). Across the two study groups, it was found that $87.2 \%$ of the subjects self-medicated due to different illnesses without prescription and procured medicines without consulting a doctor in the last 4 months. The socio- demographic profile of nonprescription medicine users is shown in Table 1. The percentages of different medicines that were dispensed without prescription in the last 4 months are shown in the Table 2 . Table 3 reveals the class of that were dispensed medicines from the community pharmacy and the hospital PPP pharmacy, where a significant difference was noted only for the eye drops $(p=0.003)$. The maximum number of encounters in the last 4 months was 8 . Again, 104 out of 392 study subjects selected the antibiotics without prescription for their ailments. The knowledge of antibiotics being limited, recommendation from the dispenser was the most common motivation (28.8\%), followed by past experiences (21\%), opinion of family members and relatives(20\%), previous prescriptions for them/ others(17\%), opinion from friends(10\%). Recommendation by internet users is the least common source of antibiotic users (1\%). Figure 1 shows the different indications of non-prescription antibiotics, the most common being fever, sore throat, and diarrhoea. The preferred source of antibiotics were medicine shops followed by left over strips from previous prescription. Majority of them preferred 4 types \& 3 types $(37.5 \% \& 28.9 \%$ respectively) of antibiotics in the form of self-medication for a single episode of illness in last 3 months. The knowledge of the required antibiotic dose for a single episode of illness varied widely as shown in Figure 2. Only $20.4 \%$ of total non-prescription medicine users were aware of the fact that same medicines may be sold with different brand names. Figure 3 shows that more than half of the subjects (57.9\%) discontinued the intake of antibiotics on subsidence of their symptoms while only few (12.5\%) of them stopped the treatment only after completion of the course of antibiotics. Figure 4 shows various types of remedy taken by the study subjects to manage their side effects, of which consulting a pharmacy staff or 
Table 1: Basic socio-demographic characteristics of the non-prescription medicine users $(n=392)$

\begin{tabular}{ccc}
\hline Parameter & Percentage & p-value* \\
\hline Age (years) & 26 & 0.6 \\
$23-44$ & 74 & \\
$>44$ & & 0.6 \\
Gender & 70.2 & \\
Male & 29.8 & 0.7 \\
Female & & \\
Residence & 55.1 & \\
Urban & 41.8 & 0.2 \\
Semi-urban & 3.1 & \\
Rural & & \\
Education & 63.8 & \\
Higher secondary & 36.2 & \\
Secondary or low & & \\
Monthly income & 56.4 & \\
$>$ rupees 19574 & 7.1 & \\
9788-19574 & 3.6 & \\
7323-9787 & 15.6 & \\
$4894-7322$ & 11.5 & \\
2936-4893 & 5.9 & \\
980-2935 & & \\
\hline
\end{tabular}

${ }^{*} \mathrm{p}$-value obtained by the application of Chi square test using SPSS Version 20 software.

Table 2: Classes of medicines procured without prescription ( $n=392)$

\begin{tabular}{ccc}
\hline Drug groups & Frequency & Percentage \\
\hline Antibiotics & 104 & 26.5 \\
Analgesics \& anti-inflammatory agents & 167 & 42.6 \\
Antiulcer agents & 170 & 43.4 \\
Antiemetics & 59 & 15.1 \\
Laxatives & 76 & 19.34 \\
Anti-diarrhoeals & 131 & 33.4 \\
Antihistamines & 13 & 3.3 \\
Antitussives & 59 & 15.1 \\
Vitamin and nutrients & 54 & 13.8 \\
Sedatives and anxiolytics & 1 & 0.3 \\
Anti-depressants & 4 & 1 \\
Eye drops & 19 & 4.8 \\
pral contraceptives \& "morning after" & 24 & 6.1 \\
inhalers \& rotahalers & 32 & 8.2 \\
\hline
\end{tabular}

person at the dispensing counter was felt easier than consulting a physician. It was observed that $167(42.6 \%)$ non-prescription medicine users preferred antipyretic-analgesic fixed dose combination (FDC) including paracetamol mainly for fever (93\%). Only $19 \%$ of them consumed such FDCs for inflammatory conditions. Consumption of ORS was common for diarrhoea as compared to vomiting. Only $13 \%$ \& $11 \%$ of the respondents dissolved the ORS powder in one litre of water in episodes of diarrhoea \& vomiting respectively. It was observed that 10 out of $13(76.9 \%)$ anti-allergic medicine users reported of drowsiness. Lastly among the all non prescription medicine users, $64.5 \%$ had a firm belief

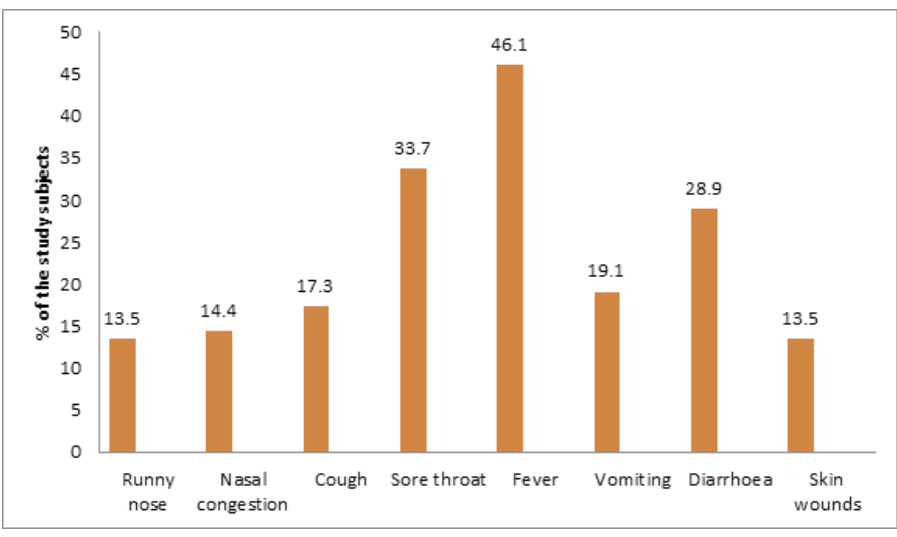

Figure 1: Clinical conditions that required the self-medication with antibiotics $(n=104)$.

\section{Knowledge of the intended dose of antibiotics for practicing self-medication $(n=104)$}

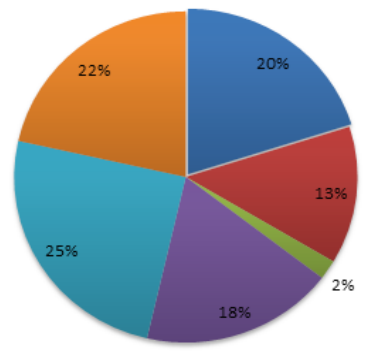

Consulting a pharmacist

Information from prevoius consultation with a clinician

E Information from the package insert of the oral antibiotic Consult ing family members or friends

Experience from previous episode of illness

Applying own knowledge to decide the right dose

Figure 2: Knowledge of the intended dose of antibiotics for practicing selfmedication $(n=104)$.

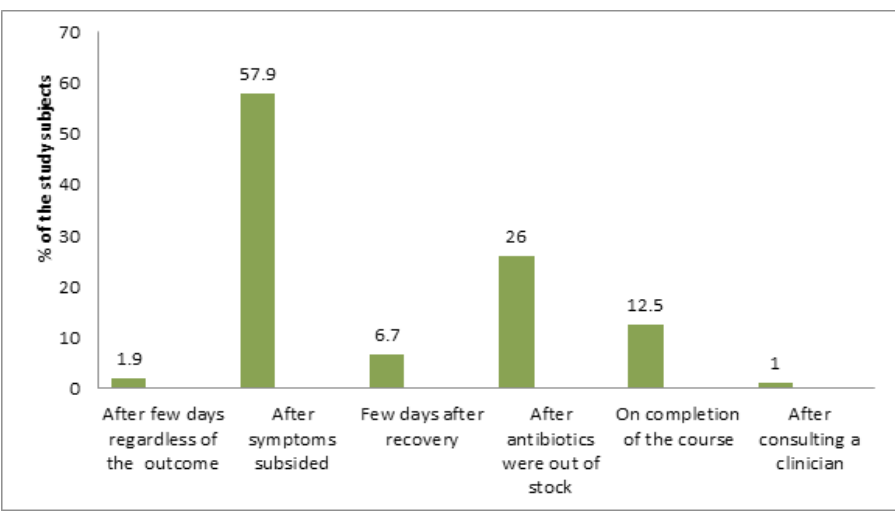

Figure 3: Reasons for discontinuation of the course of antibiotics ( $n=104)$.

that self- medication is an acceptable practice, $25.8 \%$ thought it a good practice and only $9.7 \%$ of them agreed that such practice is not acceptable.

\section{DISCUSSION}

Self-medication is widely practiced worldwide and often considered as a component of self-care. We found that across the two study groups, a majority i.e about $87 \%$ preferred self-medication practice due to various ailments for themselves, family members, relatives and friends in the last 4 months. This study revealed that not less than $61.4 \%$ of the medicine 
Table 3: Consumption pattern of non-prescription medicines from the community and PPP hospital pharmacy counters ( $n=392)$

\begin{tabular}{cccc}
\hline Drug groups & $\begin{array}{c}\text { No. of consumers from } \\
\text { community retail outlet }\end{array}$ & $\begin{array}{c}\text { No. of consumers from the } \\
\text { hospital pharmacy }\end{array}$ & p-value \\
\hline Antibiotics & 70 & 34 & 0.5 \\
Analgesics \& anti-inflammatory agents & 104 & 63 & 0.9 \\
Antacids \& antiulcer agents & 110 & 60 & 0.5 \\
Antiemetics & 36 & 23 & 0.7 \\
Laxatives & 51 & 22 & 0.1 \\
Anti-diarrhoeals & 77 & 56 & 0.1 \\
Antihistamines & 8 & 5 & 1.0 \\
Antitussives & 37 & 22 & 0.0 \\
Multivitamins & 35 & 19 & 1.0 \\
Sedatives \& anxiolytics & 1 & 0 & 0.3 \\
Anti-depressants & 4 & 0 & 0.003 \\
Eye drops & 16 & 3 & 0.6 \\
Oral contraceptives \& "morning after" pills & 14 & 10 & 0.09 \\
\hline Inhalers \& rotahalers & 21 & 11 & 0.0 \\
\hline
\end{tabular}

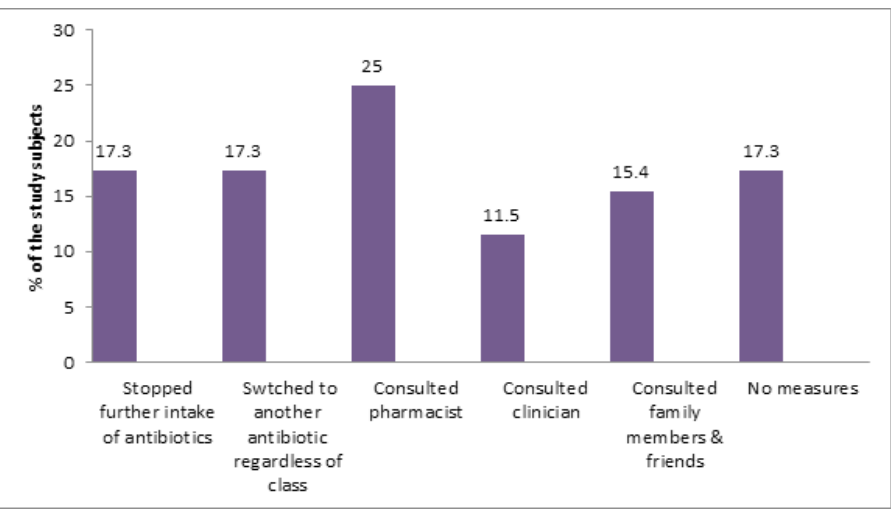

Figure 4: Remedy opted for the management of side effects due to selfmedication with antibiotics $(n=104)$.

consumers indulged in self-medication practice in the community and greater proportion of the beneficiaries depended on the community based retail medicine shop. It was seen that there were various reasons for preferring self-medication practice in the study subjects. More than one third of them (35.7\%) preferred self-medication as it was a common practice in their locality and almost similar proportion preferred affordability $(33.7 \%)$, followed by convenience $(30.1 \%)$. Only $0.5 \%$ consumer had lack of trust to the modern medicine and the physician. This finding is in discordance with another recent survey ${ }^{5}$ that revealed common reasons for self-medication as ailments being minor $(85.2 \%)$ or prior experience with the same problem (49.5\%). A study conducted in a rural area of north India ${ }^{15}$ has shown self-medication with NSAIDs alone or in FDCs (25.3\%), GI medicines (20.8\%) and antibiotics (16.7\%) while we found self-medication in $43.4 \%$ of the study subjects with antacids followed by antipyretic-analgesic including paracetamol(42.6\%) alone and in FDCs. Again majority of them preferred (46.1\%) antibiotics for fever, followed by $33.7 \%$ for sore throat, $14.4 \%$ for nasal congestion, $13.5 \%$ for runny nose, and $28.9 \%$ for diarrhea. This finding corroborates with that of a study from China ${ }^{8}$ with self-medication for sore throat (59.7\%), fever (38.2\%), cough (37.4\%), runny nose (29.3\%), and nasal congestion (28.7\%). Majority of our study subjects (28.8\%) selected the antibiotics on recommendation from dispensing counter, and $21.2 \%$ from past experiences and left-over strips. This observation correlates with two recent studies ${ }^{9-16}$ where $67.3 \%$ subjects procured their medicines from retail medicine shops and the rest from left over medicines. Another study from outside India ${ }^{7}$ reported ADRs with self-medication in $16.7 \%$ of the respondents, while in our study $13.3 \%$ of the subjects reported the ADRs. In the same study $36.5 \%$ subjects switched over to another antibiotic but in our study $17.3 \%$ of the respondents switched on to another medicine during the course of antibiotics. Our study found that consumption of ORS is more common for diarrhoea than vomiting and very few (13\%) of the respondents had knowledge about the directions to dissolve and use the ORS powder. Therefore, the awareness about proper ORS usage is scarce in our study population. A study from Israel ${ }^{10}$ revealed that about $15 \%$ of the non-prescription medicine users believed that practicing self-medication is not safe whereas only $9.7 \%$ of our study subjects believed that self-medication is an unacceptable practice. In our study, individuals who were dependent on non-prescription medicine use informed us that they were never asked by pharmacy staff about their motive of procurement of medicines and this observation is in concordance with a recent survey conducted in $\mathrm{UK} .{ }^{20}$ A gross over-usage of non-prescription antibiotics, that too not taken for a full course, by most of the patients of URTI was found recently. The study has shown that lack of awareness in the patients could increase the drug resistance problem and awareness about the branded drugs being costlier than the generic drugs was also not there. ${ }^{21}$ The first Indian study which provides a comprehensive picture of self-medication practice among future healthcare providers (medical and paramedical students) from across the country shows lesser prevalence of self-medication than us. ${ }^{22}$ Limitations of this study are that we could not find an influence of the demographic profile of the subjects upon the practice of non-prescription medicine resulting in self-medication practice. The class of medicines procured from the community pharmacy and the hospital PPP pharmacy was statistically significant only for ophthalmic eye drops. This may be due to a higher prevalence and seasonal variation of ocular infections in the community from where we have selected our study group. It could also be due to ignorance of the study subjects to visit the Ophthalmology OPD of the neatest government or private hospital. Stratification of the 
study subjects according to their respective clinical specialities has not been possible in this study. Future surveys with larger sample size may be undertaken to overcome the limitations of our study.

\section{CONCLUSION}

This study has documented the extent of non-prescription medicine use, the factors associated with such an irrational practice and the pattern of such consumption of medicines that have resulted in wider and indiscriminate self-medication practice among the study subjects. However, the determinants of such practice were not elicited within the ambit of our findings and analysis. Further studies may be undertaken with greater sample stratifications to generate more interesting findings.

\section{ACKNOWLEDGEMENT}

The authors acknowledge the patients, their near keens, relatives, and friends without whose cooperation conduct of this survey would not be feasible and possible.

\section{SOURCE OF FUNDING}

Nil

\section{CONFLICT OF INTEREST}

The authors declare no conflict of interest

\section{CONTRIBUTIONS OF AUTHORS}

MD, SC and RSD planned and conceptualised this study, RKR complied with the proposal and revised further for conducting it, $\mathrm{RD}$ along with MD and SC performed the field survey, SC analysed the data, MD and RSD drafted the manuscript and all the authors finalised it for submission.

\section{REFERENCES}

1. Montastruc JL, Bagheri H, Geraud T, Lapeyre-Mestre M. Pharmacovigilance of self-medication. Therapie. 1996;52(2):105-10.

2. Hughes CM, McElnay JC, Fleming GF. Benefits and risks of self-medication. Drug safety. 2001;24(14):1027-37.

3. World Health Organization: The role of the pharmacist in self care and self medication. Available from: http://www.apps.who.int/ medicinedocs/pdf/ whozip32.pdf

4. Hughes CM, McElnay JC, Fleming GF. Benefits and risks of self-medication. Drug Saf. 2001;24(14):1027-37.

5. Al-Ramahi R. Patterns and attitudes of self-medication practices and possible role of community pharmacists in Palestine. Int $\mathrm{J}$ Clin Pharmacol Ther.
2013;51(7):562-7.

6. Osemene KP, Lamikanra A. A study of the prevalence of self-medication practice among university students in Southwestern Nigeria. Trop J Pharm Res. 2012;11(4):683-9.

7. Lv B, Zhou Z, Xu G, Yang D, Wu L, et al. Knowledge, attitudes and practices concerning self-medication with antibiotics among university students in western China. Trop Med Int Health. 2014;19(7):769-79.

8. Pan H, Cui B, Zhang D, Farrar J, Law F, et al. Prior knowledge, older age, and higher allowance are risk factors for self-medication with antibiotics among university students in southern China. PloS one. 2012;7(7):e41314

9. Grigoryan L, Monnet DL, Haaijer-Ruskamp FM, Bonten MJ, Lundborg S et al. Self-medication with antibiotics in Europe: a case for action. Curr Drug Saf. 2010;5(4):329-32.

10. Vaknin S, Abadi-Korek I, Marom E, Shemer J, Luxenburg O. The over the counter drugs reform in Israel--two years later. Harefuah. 2011;150(1):4-8.

11. Anraku M, Inoue H, Sato E, Hata T, Tsuchiya D, et al. Surveillance study in collaboration with a university-daycare center for elderly people and nursery school for children on the use of over-the-counter drugs and health food in Fukuyama. Yakugaku zasshi: Yakugaku Zasshi. 2010;130(8):1093-103.

12. Nielsen MW, Hansen EH, Rasmussen NK. Prescription and non-prescription medicine use in Denmark: association with socio-economic position. European J Clin Pharmacol. 2003;59(8-9):677-84.

13. Yuefeng $L$, Keqin R, Xiaowei R. Use of and factors associated with self-treatment in China. BMC Pub Health. 2012;12:995.

14. Ge S, He TT, Hu H. Popularity and customer preferences for over-the-counter Chinese medicines perceived by community pharmacists in Shanghai and Guangzhou: a questionnaire survey study. Chin Med. 2014;9:22.

15. Ahmad A, Patel I, Mohanta GP, Balkrishnan R. Evaluation of self-medication practices in rural area of town Sahaswan at Northern India. Ann Med Health Sci Res. 2014;4(8):73-8

16. Shehnaz SI, Agarwal AK, Khan N. A systematic review of self-medication practices among adolescents. J Adolesc Health. 2014;55(4):467-83.

17. Dineshkumar $B$, et al. Profile of medicine use in urban and rural India. Pharmacoeconomics 1995;7:332-46.

18. Watson MC, Johnston M, Entwistle V, Lee AJ, Bond CM, et al. Using the theory of planned behaviour to develop targets for interventions to enhance patient communication during pharmacy consultations for non-prescription medicines. Int J Pharm Pract. 2014;22(6):386-96.

19. Fingleton NA, Watson MC, Duncan EM, Matheson C. Non-prescription medicine misuse, abuse and dependence: a cross-sectional survey of the UK general population. J Public Health. 2016:fdv204.

20. Wazaify M, Shields E, Hughes CM, McElnay JC. Societal perspectives on overthe-counter (OTC) medicines. Fam Pract. 2005;22(2):170-6.

21. Bhanwra S. A study of non-prescription usage of antibiotics in the upper respiratory tract infections in the urban population. J Pharmacol Pharmacother. 2013;4(1):62-4.

22. Kumar R, Goyal A, Padhy BM, Gupta YK. Self-medication practice and factors influencing it among medical and paramedical students in India: A two-period comparative cross-sectional study. J Nat Sc Biol Med. 2016;7(2):143-8.

Article History: Submission Date : 20-01-2017 ; Revised Date : 04-05-2017; Acceptance Date : 11-06-2017.

Cite this article: Das M, Choudhury S, Santra R (Dhali), De R, Roy RK. The Extent and Factors Associated with Non-Prescription Medicine Use in Eastern India - A Cross-Sectional Survey. JYoung Pharm. 2017;9(4):583-7. 